\title{
How the planetary research helps to the stellar dynamo understanding
}

\author{
I. Boisse ${ }^{1}$, M. Oshagh ${ }^{1,2}$, C. Lovis ${ }^{3}$, N. C. Santos ${ }^{1,2}$, X. Dumusque ${ }^{2,3}$, \\ X. Bonfils ${ }^{4}$, M. Montalto ${ }^{1}$ and G. Boué ${ }^{1}$ \\ ${ }^{1}$ Centro de Astrofísica, Universidade do Porto, Rua das Estrelas, 4150-762 Porto, Portugal \\ email: Isabelle.Boisse@astro.up.pt \\ ${ }^{2}$ Departamento de Física e Astronomia, Faculdade de Ciências, Universidade do Porto, \\ 4169-007 Porto, Portugal \\ ${ }^{3}$ Observatoire de Genève, Université de Genève, 51 Ch. des Maillettes, 1290 Sauverny, \\ Switzerland \\ ${ }^{4}$ UJF-Grenoble1/CNRS-INSU, Institut de Planétologie et d'Astrophysique de Grenoble, UMR \\ 5274, Grenoble, F-38041, France
}

\begin{abstract}
Most of the exoplanet science is dependent on the stellar knowledge. One of them that has to be understood is the magnetic activity when we search for planets with radial velocity or photometry measurements. The main shape of stellar activity and spots properties have to be understood, for example, to choose the best targets to search for low-mass planets in the habitable zone or to derive the accurate parameters of a planetary system. With that aim, we show in this presentation how these studies lead to give clues on spots latitudes and on the long term variation of stellar activity. The properties of magnetic activity on the low rotators solar-type stars are not easily reachable by other techniques (spectropolarimetry or Doppler imaging) and these studies should be used to constrain theories of stellar dynamo.
\end{abstract}

Keywords. Techniques: radial velocities, photometry, Stars: activity, Dynamo, Planetary system

\section{Why planetary researchers are worried about stellar activity?}

Up to now, more than 800 planets were discovered and characterized, most of them thanks to the radial-velocity (RV) technique. However, this efficient method is indirect as well as the photometric transit and astrometry techniques. One of the problems with this is the fact that RV variations can in some cases be caused by other mechanisms that are not related to the presence of low-mass companions. Even if RV surveys could reject very active targets, all stars present variability at different level (from few $\mathrm{cm}^{-1} \mathrm{~s}$ to hundreds of $\mathrm{m}^{-1} \mathrm{~s}$ ). Moreover, mainly RV is needed to establish the planetary nature and measure the mass of a transiting candidate (and stellar activity is less determinant for transit detection).

RV values are derived from the measurement of position in wavelength of thousands of spectral lines. Unfortunately, the measurement is not only sensitive to Doppler shift due to gravitationally bounded companion but also to line profile deformations. Especially, apparition and disappearance of spots from the observer view due to the stellar rotation, or change of the convection pattern with magnetic cycle induce a distortion of the line that changes with time. The jitter that results in RV measurement may hamper planet discovery or degrade the orbital parameters estimation. They may also give false candidates, if they produce a stable periodic signal (e.g. Queloz et al. 2001). An essential work is then needed to understand all phenomena caused by stellar variability, and to 
characterize their impact on RV and photometry to be able to distinguish between these and real planetary signatures.

\section{How planetary researchers track stellar activity?}

Phenomena such as stellar pulsation or inhomogeneous convection could be averaged by specific observational strategy (Dumusque et al. 2011). Because the typical timescale of those oscillations is less than fifteen minutes, an exposure time of fifteen minutes or larger reduces greatly the oscillation noise. In the same way, the timescale of granulation is less than 30 hours, therefore taking several measurements per night allows a reduction of the RV contribution of granulation. These methods have already been used by Pepe et al. (2011) and showed good efficiency (e.g. Santos et al. 2004, that averaged asteroseismologic data to detect one of the first low-mass planet).

In the following, we focus on stellar activity, mainly dark spots and bright plages on the stellar surface, and on magnetic cycle. These phenomenon are assumed to be part of the stellar dynamo process, that generates stellar magnetic field. To identify stellar activity, we can monitor the bisector span (BIS), the width and the depth of the mean line of the spectrum, and also calculate the chromospheric emission ratio, $\log \mathrm{R}_{\mathrm{H} K}^{\prime}$ from the $\mathrm{CaII} H \& \mathrm{~K}$ lines and monitor other lines sensitive to active features: $\mathrm{H} \alpha$, HeI, NaI. However the behavior of these parameters is not yet understood properly, moreover because they depend on stellar parameters (Desort et al. 2007, Boisse et al. 2011, Boisse et al. 2012). For example, Bonfils et al. (2007) has to compared RV and stellar activity index, because the BIS becomes inefficient as an indicator of activity when the star rotates too low.

\section{SOAP: a tool for the fast computation of the stellar spots impact $\dagger$}

SOAP is an online software that simulates the effect of stellar spots and plages on RV and photometry (Boisse et al. 2012). This numerical model centers the visible stellar disk on a grid of several tens of thousands of resolution elements. The typical line of the emerging spectrum of the star is modeled by a Gaussian for each grid cell (equivalent to the spectrum cross-correlation function that can be seen as a weighted average of all the spectral lines convolved with a rectangular function). Each Gaussian (in a given stellar position) is Doppler-shifted according to the projected rotational velocity and weighted by a linear limb-darkening law. SOAP calculates the position of the surface inhomogeneities defined by their latitudes, longitudes, and sizes. The cells inside the spots are modeled by the same Gaussian as for the stellar disk, but are weighted by their brightness. The code then removes (for dark spot) or adds (for bright plage) the Gaussian and flux of the inhomogeneities to those of the non-spotted star. Finally, SOAP delivers the integrated spectral line, the flux, the RV, and the bisector span as a function of the stellar rotational phase.

SOAP-T is an improved version that includes the impact of a transiting planet in RV and photometry (Oshagh et al. 2012).

\section{What we learnt about stellar activity?}

Monitoring and determining properties of active regions

Boisse et al. (2009) monitored HD189733 simultaneously in RV with SOPHIE and in photometry with the MOST satellite. They showed the presence of an active region on

$\dagger$ The tool is available at http://www.astro.up.pt/soap 
the stellar surface. The plage was tracked by $\mathrm{H} \alpha$ emission, but in photometry the dark spot dominates the signal. The spots temperature were constrained from the crossing of the transiting planet observed by HST (Pont et al. 2007). The ratio of the covered surface by dark spots and bright plages, and their contrast in temperature with the stellar photosphere, are key parameters to model activity, mainly to analyze photometric rotational modulations from Kepler and CoRoT lightcurves (e.g. Lanza et al. 2011, Kipping 2012).

\section{Long-term $R V$ and $\log R_{\mathrm{H} K}^{\prime}$ variations: Characterizing photospheric modifications with magnetic field evolution}

RV surveys searching for planets monitor primarily main-sequence stars of spectral-type from A-F to M. Some database reach now a time coverage of more than 15 years. Even if the sampling of the observation and the target selection are not optimized for activity monitoring, results can be drawn about long term variations.

Lovis et al. (2011) studied 304 FGK stars, mainly dwarfs, from the HARPS sample. They searched for long term variability with period greater than 2 years. They computed the activity index, $\log \mathrm{R}_{\mathrm{H} K}^{\prime}$, from the CaII H\&K lines. They compared its variability with the one of the RV, BIS and parameters of the mean spectral line. We would like to point out three of their results. They detected variability periods shorter than the 11 yrs of the Sun cycle. It may be a sign of multiperiodism in other stars. They found few F stars present periodicity. But we could also argue that magnetic cycle measured in F stars (Tau Boo Farès et al. 2009, Iota Hor Metcalfe et al. 2010) present periods shorter than 2 years. The last point is the dependence of the correlation slope between RV and $R_{\mathrm{H} K}^{\prime}$, as a function of effective temperature (see Fig. 1). This indicate that for a same variation in the $\log \mathrm{R}_{\mathrm{H} K}^{\prime}$, the impact in $\mathrm{RV}$ will be 2-3 times greater for a $\mathrm{G}$ dwarf than for a late $\mathrm{K}$ star. Which phenomena (or composition of phenomena) is responsible for this is still an open question. It could be due to a different modification of the convection pattern with activity level or to a distinct filling factor of plages compared to spots with spectral types.

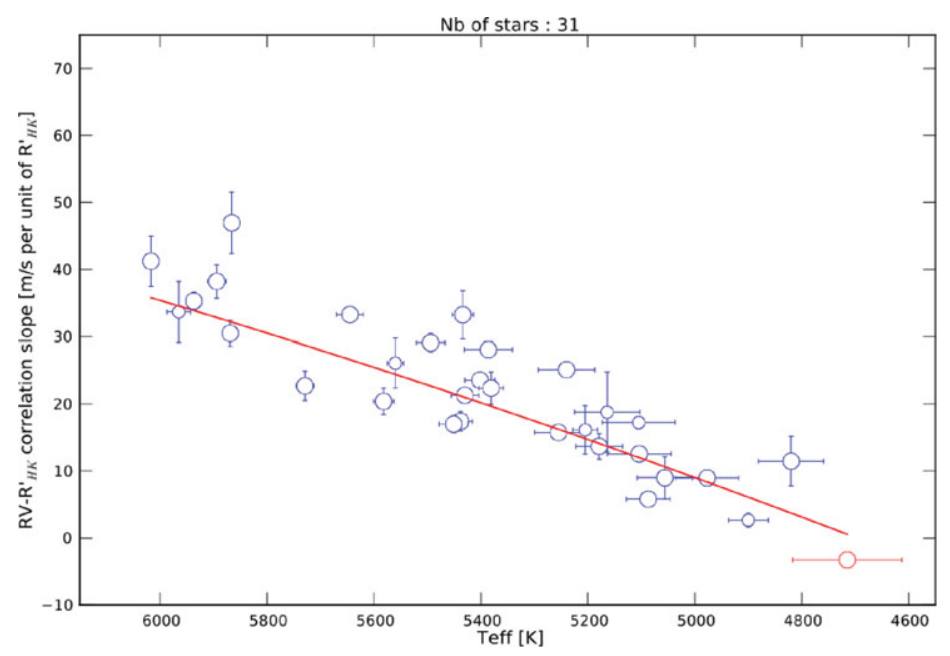

Figure 1. Correlation slope between RV and $R_{\mathrm{H} K}^{\prime}$, as a function of effective temperature (from Lovis et al. 2011). 


\section{Track the spots with planetary transits}

A planet transiting its host-star could overlap with active zones on the stellar surface. It will generate anomalies (bumps) inside the transit light-curve (see Fig. 2). The evolution of these anomalies can be tracked if long-term and continuous observations are accessible (e.g. CoRoT and Kepler). It could allow to determine the spin-orbit angle, the inclination of the stellar spin axis, and the configuration of the spots on the stellar surface (e.g. Nutzman et al. 2011, Désert et al. 2011, Sanchis-Ojeda et al. 2011.

Sanchis-Ojeda \& Winn (2011) detected two bumps in the folded Kepler light-curve of 26 transits of HAT-P-11. They interpreted these peaks as evidence of long lived spot belt regions, and derived two possible scenario for the planet orbit, stellar spin axis and spots positions. Using SOAP-T and the out-of-transit light curve, Oshagh et al. (2012) showed that the most probable scenario of the previous study is the one that presents two active belts at $\sim \pm 20^{\circ}$, comparable to what is observed on the Sun but at lower latitude. Kepler will continue to monitor the star during several years. In analogy to what is seen on the Sun, we could expect to observe a change in the spots latitude with time if HAT-P-11 has a magnetic cycle.
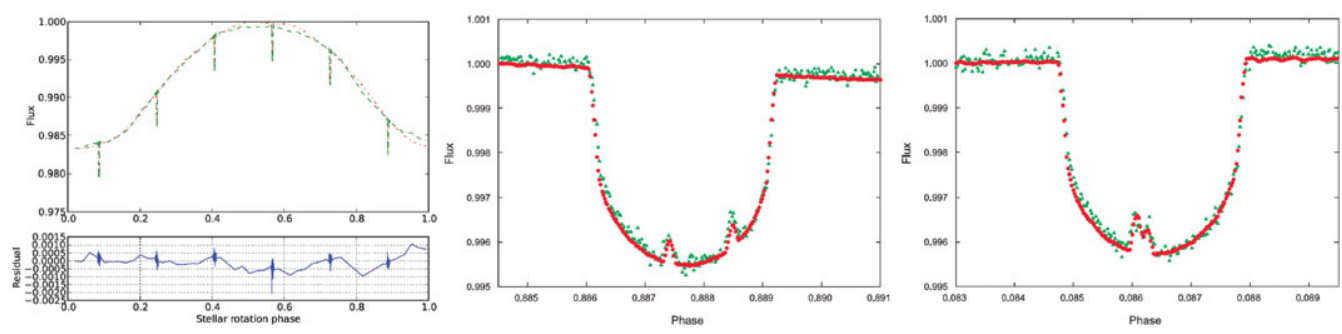

Figure 2. Left: Kepler observation of HAT-P-11 (green) and SOAP-T fit (red) of HAT-P-11 during one stellar rotation. Blue are the residuals. Middle and right: Zooms on two transits of the left figure. The flux values are normalized to one. Figures from Oshagh et al. (2012).

\section{Future}

SOAP simulates the photometric effect due to inhomogeneities on the stellar surface. However, it does not consider the effect that they also block the convection pattern. Moreover, spots and plages are not only areas darker or brighter, but have a different temperature (then a different spectra) than the stellar photosphere.

The wavelength dependency of spots must be taken into account because, if most of the accurate spectrographs are now in the optical, soon near-infrared instruments will be available, a wavelength domain in which the amplitude of RV variation due to activity is smaller (e.g. Huélamo et al. 2008). Stellar magnetic fields might be monitored by polarimetry of the stellar lines induced by Zeeman effect with instruments such as ESPADONS@CFHT, Narval@TBL, HARPSPol@ESO, or soon SPIRou@CFHT also dedicated to the search of planets around low-mass stars.

Dedicated observations of active stars are therefore needed, if possible simultaneously, in RV, photometry, and polarimetry to characterize the different level of stellar variability, to understand the correlation between the different proxies, and to constrain the simulations. The release of thousands of light curves from the space missions CoRoT and Kepler dedicated to asteroseismology and the search for planets via transit will help us to understand statistically the photometric variability of these stars. 


\section{Acknowledgements}

IB, MO, NCS, MM, and GB acknowledge the support of the European Research Council/ European Community under the FP7 through a Starting Grant, as well from Fundaccao para a Ciência e a Tecnologia (FCT), Portugal, through SFRH/BPD/81084/2011 and the project PTDC/CTE-AST/098528/2008.

\section{References}

Boisse, I., Moutou, C., Vidal-Madjar, A. et al. 2009, A\&A, 495, 959

Boisse, I., Bouchy, F., Hébrard, G. et al. 2011, A\& $A$, 528, A4

Boisse, I., Bonfils, X., \& Santos, N. C. 2012, A\&A, 545, 109

Bonfils, X., Mayor, M., Delfosse, X. et al. 2007, A\& $A, 474,293$

Désert, J.-M., Charbonneau, D., Demory, B.-O. et al. 2011, ApJS, 197, 14

Desort, M., Lagrange, A;-M., Galland, F., Udry, S.,\& Mayor, M. 2007, A\&A, 473, 983

Dumusque, X., Udry, S., Lovis, C., Santos, N. C., \& Monteiro, M. 2011, A\&AA, 525, 140

Farès, R., Donati, J.-F., \& Moutou, C. et al. 2009, MNRAS, 398, 1383

Huélamo, N., Figueira, P., Bonfils, X. et al. 2008, A $\mathscr{E} A$, 489, L9

Kipping 2012, arXiv: 1209.2985

Lanza, A. F., Boisse, I., Bouchy, F., \& Bonomo, A. S. and Moutou, C. 2011, A\& A, 533, 44

Lovis, C., Dumusque, X., \& Santos, N. C. et al. 2011, arXiv:1107.5325

Metcalfe, T. S., Basu, S., Henry, T. J. et al. 2010, ApJ, 723, 213

Nutzman, Fabrycky \& Fortney 2011, ApJ, 740, L10

Oshagh, M., Boisse, I., Boué, G. et al. 2012, A\&A, in press

Pepe, F., Lovis, C., Ségransan, D. et al. 2011, A\& $A, 534 \mathrm{~A}, 58 \mathrm{P} 1$

Pont, F., Gilliland, R. L., Moutou, C. et al. 2007, $A \& A, 476,1347$

Queloz, D., Henry, G. W., Sivan, J.-P. et al. 2001, A\&A, 379, 279

Sanchis-Ojeda, R., Winn, J. N., Holman, M. et al. 2011, ApJ, 733, 127

Sanchis-Ojeda \& Winn 2011, ApJ, 743, 61

Santos, N. C., Bouchy, F., Mayor, M. et al. 2004, A\&\&A, 426L, 19 